\title{
A Large-scale Comparison of Cortical and Subcortical Structural Segmentation Methods in Alzheimer's Disease: a Statistical Approach
}

\author{
Jafar Zamani', Ali Sadr ${ }^{1, *}$, Amir-Homayoun Javadi ${ }^{2,3,4, *}$
}

${ }^{1}$ School of Electrical Engineering, Iran University of Science and Technology, Tehran, Iran

${ }^{2}$ School of Psychology, University of Kent, Canterbury, UK

${ }^{3}$ Institute of Behavioural Neuroscience, University College London, London, UK

${ }^{4}$ School of Rehabilitation, Tehran University of Medical Sciences, Tehran, Iran

Running title: Structural Segmentation in Alzheimer's Disease

\section{*Corresponding authors:}

\author{
Ali Sadr \\ Address: $\quad$ School of Electrical Engineering, \\ Iran University of Science \& Technology, \\ Narmak, Tehran, Iran \\ Phone: $\quad+982173225757$ \\ E-Mail:_sadr@iust.ac.ir \\ Address: $\quad$ School of Psychology, Keynes College \\ University of Kent \\ Canterbury, UK \\ Phone: $\quad$ +44 1227827770 \\ E-Mail: $\quad$ a.h.javadi@gmail.com
}




\section{Abstract}

Background. Alzheimer's disease $(\mathrm{AD})$ is a neurodegenerative disease that leads to anatomical atrophy, as evidenced by magnetic resonance imaging (MRI). Automated segmentation methods are developed to help with the segmentation of different brain areas. However, their reliability has yet to be fully investigated. To have a more comprehensive understanding of the distribution of changes in $\mathrm{AD}$, as well as investigating the reliability of different segmentation methods, in this study we compared volumes of cortical and subcortical brain segments, using automated segmentation methods in more than 60 areas between AD and healthy controls (HC).

Methods. A total of $44 \mathrm{MRI}$ images (22 AD and $22 \mathrm{HC}, 50 \%$ females) were taken from the minimal interval resonance imaging in Alzheimer's disease (MIRIAD) dataset. HIPS, volBrain, CAT and BrainSuite segmentation methods were used for the subfields of hippocampus, and the rest of the brain.

Results. While HIPS, volBrain and CAT showed strong conformity with the past literature, BrainSuite misclassified several brain areas. Additionally, the volume of the brain areas that successfully discriminated between $\mathrm{AD}$ and $\mathrm{HC}$ showed a correlation with mini mental state examination (MMSE) scores. The two methods of volBrain and CAT showed a very strong correlation. These two methods, however, did not correlate with BrainSuite.

Conclusion. Our results showed that automated segmentation methods HIPS, volBrain and CAT can be used in the classification of $\mathrm{AD}$ and $\mathrm{HC}$. This is an indication that such methods can be used to inform researchers and clinicians of underlying mechanisms and progression of AD.

Keywords: volumetric MRI T1, atrophy, automatic segmentation, dementia, volBrain, CAT, BrainSuite, HIPS 


\section{Introduction}

Alzheimer's disease (AD) is a devasting neurodegenerative disease, contributing to $60-70 \%$ of dementia cases ${ }^{1}$. Currently there are around 50 million people with dementia worldwide. In 2015 , the total global societal cost of dementia was estimated to be US $\$ 818$ billion ${ }^{2,3}$. Mainly due to increased life-expectancy, the total number of people with dementia is projected to reach 82 million (64\% increase) in 2030, with between 49 and 57 million of these cases being $\mathrm{AD}^{4}$. Whilst several drugs are available to mitigate the symptoms in some cases, no treatments are available that prevent progression from the relatively late stage at which the disease is diagnosed ${ }^{5}$.

$\mathrm{AD}$ is characterised by two main pathological hallmarks: extracellular amyloid deposits, composed of insoluble amyloid beta $(\mathrm{A} \beta)$ protein, and intra-neuronal neurofibrillary tangles (NFTs), containing hyperphosphorylated tau protein ${ }^{6}$. AD is also characterised by a significant loss of neurons and synapses, resulting in brain shrinkage and atrophy ${ }^{7,8}$. Structural changes have been shown to be one of the earliest biomarkers that can be used in the diagnosis of AD 9-11. Much effort has been devoted to find patterns of changes in the structure of different brain areas that can be reliably used for diagnosis of $\mathrm{AD}^{12}$.

Earlier investigations relied mostly on manual segmentation of brain areas requiring a great deal of expertise and time ${ }^{13-16}$. Therefore, the majority of the focus has been devoted to changes in the hippocampus due to its distinct structure ${ }^{17}$. It has been shown that a loss in hippocampal volume can be an indication of $\mathrm{AD}{ }^{18,19}$. Further investigations have looked at subfields of the hippocampus, showing a nonuniform rate of neuroplasticity due to their specialisation ${ }^{20-22}$. For example, it has been shown that NFT begin in the medial temporal region and exhibit a characteristic distribution pattern across subfields, starting in the CA1 and later spreading to subiculum, CA2, CA3 and CA4/Dentate Gyrus ${ }^{23-27}$.

With the development of semi- and fully-automated segmentation methods, however, it has now become easier and faster to segment not only the hippocampal area, but also other brain areas ${ }^{28-32}$. HIPpocampus subfield Segmentation (HIPS) ${ }^{33}$, volBrain ${ }^{34}$, Computational Anatomy Toolbox (CAT) ${ }^{35,36}$, BrainSuite ${ }^{37,38}$ and FreeSurfer ${ }^{39}$ are some of the commonly used semi- and fully-automated methods. These methods, however, are yet under development 40,41. For example, CA1 segmentation in the FreeSurfer v5.3 was partially included in the subiculum ${ }^{18}$ potentially explaining why the CA1 field was reported to be insensitive to AD pathology in some ${ }^{42,43}$ but not all ${ }^{44,45}$. Similar findings have recently raised questions and 
concerns regarding the accuracy and consistency of these methods ${ }^{46-49}$. Therefore, it is important to investigate the accuracy of these methods further ${ }^{50}$.

Benefiting from the computational power of automated methods, analysis of a large number of brain images has become more feasible. Large datasets of brain scans such as Minimal Interval Resonance Imaging in Alzheimer's Disease (MIRIAD), a public database of Alzheimer's magnetic resonance imaging (MRI) ${ }^{51}$, offer a great opportunity to have a more comprehensive approach to the underlying mechanism and progression of $\mathrm{AD}^{52-56}$. It also facilitates multisite studies to form a more accurate understanding of the disease ${ }^{57,58}$.

Mini mental state examination (MMSE) is one of the commonly accepted measurements of cognitive ability, in particular in clinical settings ${ }^{59-61}$. This measure has been widely used in classification of AD. For example, MIRIAD classifies participants with score between 12 and $26 / 30$ as $\mathrm{AD}$ and those higher than $26 / 30$ as healthy control. There is huge body of literature showing correlation between MMSE score and brain atrophy ${ }^{62-64}$.

The aim of this study was to investigate the reliability of four automated segmentation methods of volBrain, CAT and BrainSuite for segmentation of the whole brain, and HIPS for segmentation of subfields of hippocampus, which belongs to the same analysis tool as volBrain. We used images belonging to MIRIAD. Correlation of the volume of each brain area with MMSE scores are also investigated. To investigate the reliability of the three methods volBrain, CAT and BrainSuite, the correlation of their common brain areas is also reported.

\section{Material and Methods}

\subsection{Subjects}

We used 44 images (22 AD and $22 \mathrm{HC}$ ) taken from the Minimal Interval Resonance Imaging in Alzheimer's Disease (MIRIAD) dataset ${ }^{51}$. Table 1 shows a summary of the descriptives of the participants.

\subsection{Magnetic Resonance Imaging (MRI)}

Data was extracted from MIRIAD database. All subjects underwent MRI scanning on a $1.5 \mathrm{~T}$ Signa scanner (GE Medical Systems, Milwaukee, WI, USA). T1-weighted volumetric images were obtained using an inversion recovery prepared fast spoiled gradient echo sequence with acquisition parameters time to repetition $=15 \mathrm{~ms}$, time to echo $=5.4 \mathrm{~ms}$, flip angle $=15^{\circ}, \mathrm{TI}=$ 
$650 \mathrm{~ms}$, a $24-\mathrm{cm}$ field of view and a $256 \times 256$ matrix, to provide 124 contiguous $1.5-\mathrm{mm}$ thick slices in the coronal plane (voxels $\left.0.9735 \times 0.9735 \times 1.5 \mathrm{~mm}^{3}\right)^{51}$.

\subsection{Methods}

HIPS and volBrain; The volumes of Cerebrospinal fluid (CSF), white matter (WM), grey matter (GM), brain hemispheres, cerebellum and brainstem were obtained using volBrain pipeline ${ }^{34}$. This method is based on an advanced pipeline providing automatic segmentation of different brain structures from T1 weighted MRI, Figure 1. The preprocessing is based on the following procedure: (1) a denoising step with an adaptive non-local mean filter, (2) an affine registration in the Montreal Neurological Institute (MNI) space, (3) a correction of the image inhomogeneities, and (4) an intensity normalisation. (5) Afterwards, MRI images are segmented in the MNI space using non-local patch-based multi-atlas method. Images were corrected for intensity inhomogeneity using the N4 algorithm ${ }^{65}$, and the images were segmented into brain/non-brain using a semi-automated technique (MIDAS). The Non-Local Means filter ${ }^{66}$ was applied to each pixel of the image by computing a weighted average of surrounding pixels using a robust similarity measure that takes into account the neighbouring pixels surrounding the pixel being compared. This segmentation method is based on the idea of non-local patch-based label fusion technique, where patches of the brain image to be segmented are compared with those of the training library, looking for similar patterns within a defined search volume to assign the proper label ${ }^{67,68}$. HIPS and volBrain are used for segmentation of the hippocampus subfields and the rest of the brain, respectively ${ }^{33}$.

CAT; Computational Anatomy Toolbox (CAT) is a powerful package for brain T1-MRI data segmentation, Figure 2. It is a voxel base estimation method ${ }^{69}$. The CAT preprocessing steps are as follows: (1) spatial registration to a template, (2) tissue segmentation into grey, white matter and CSF, and (3) bias correction of intensity non-uniformities. (4) Finally, segments are extracted by scaling the amount of volume changes based on spatial registration, so that the total volume of grey matter in the modulated image remains the same as the original image. For correction of the orientation and size of the brain, non-linear registration methods are applied to the image ${ }^{70}$. Projection-based thickness (PBT) method is used for calculation of the cortical thickness and central surface ${ }^{70,71}$. Spatial-adaptive Non-Local Means (SANLM) and classical Markov Random Field (MRF) were used for image Denoising ${ }^{72}$. Adaptive Maximum a Posterior (AMAP) method was used for segmentation ${ }^{69}$. 
BrainSuite; BrainSuite is an open source software tool that enables largely automated cortical surface extraction from MRI of the brain, Figure 3. BrainSuite includes automatic cortical surface extraction, bias field correlation, cerebrum labelling, and surface generation features. Also, this toolbox is used in tractography and connectivity matrix calculation in diffusion imaging data ${ }^{37}$.

\subsection{Statistical Analysis}

Independent-sample t-tests are run to compare the volume of different brain areas between the $\mathrm{AD}$ and HC groups for volBrain, CAT and BrainSuite for the whole brain, and HIPS for the hippocampus subfields. Bivariate-correlation analyses are also run to investigate the relationship between volume and MMSE scores for all four segmentation methods. Correlational analyses are run between the common brain areas in volBrain, CAT and BrainSuite to investigate the relationship between the three methods. Bonferroni correction is applied to account for multiple comparison.

\section{Results}

Using three automatic segmentation methods CAT, volBrain and BrainSuite, we segmented the whole brain, and using HIPS we segmented the hippocampus. Using independent-sample t-tests we compared the volumetric data for AD and HC for each segment. Figures 3-6 show sample output images for one AD patient and one HC participant. Furthermore, we investigated the correlation of volumetric data with MMSE scores in both groups.

CAT segmentation method returned data for 63 distinct brain areas. This method highlighted many brain areas that are significantly different between the two groups, Table 2. In particular fusiform gyrus, parahippocampal gyrus, hippocampus, entorhinal cortex, amygdala, temporal gyri, thalamus, nucleus accumbens, insula, caudate and precuneus were significantly different. Importantly, the size of all these brain areas showed a strong correlation with MMSE scores. For further details see supplementary figures 1-3.

$===$ Table $2===$

$===$ Figure 4 ==

volBrain segmentation method returned data for eight distinct brain areas. In particular the amygdala, hippocampus, nucleus accumbens, thalamus and caudate were significantly 
different between the two groups, Table 3. Again, the size of all these brain areas showed a strong correlation with MMSE scores. For further details see supplementary figures 4-6.

$===$ Table 3 ===

$===$ Figure $5==$

BrainSuite segmentation method returned data for 50 distinct brain areas. In contrast to CAT and volBrain, this method highlighted only six brain areas that are significantly different between the two groups, Table 4. These brain areas included temporal gyri, third ventricle, supramarginal gyrus and angular gyrus. Similar to previous segmentation methods, all these brain areas showed strong correlation with MMSE scores. For further details see supplementary figures 7-9.

$===$ Table 4 ===

$===$ Figure $6===$

HIPS segmentation method returned data for the whole hippocampus and five of its subfields: CA1, CA2-CA3, CA4/Dentate Gyrus, Subiculum and strata radiatum/lacunosum/moleculare (SR-SL-SM). All these areas showed a significant difference between the two groups, Table 5. The size of hippocampus and all its subfields showed strong correlation with MMSE scores. For further details see supplementary figures 10-12.

$===$ Table 5 ===

$===$ Figure $7===$

To investigate the relationship between the three whole-brain segmentation methods CAT, volBrain and BrainSuite, we ran correlational analysis, Table 6. Seven brain areas were common between these methods: nucleus accumbens, amygdala, caudate, globus pallidus, hippocampus, putamen and thalamus. CAT and volBrain showed strong correlation for nucleus accumbens, amygdala, caudate, hippocampus and thalamus. Two brain areas globus pallidus and putamen were not significantly correlated. These brain areas did not show significant difference between the two groups either. BrainSuite, however, showed no significant correlation with either of the other two segmentation methods.

$===$ Table $6===$ 


\section{Discussion}

We used HIPS automated method to segment the subfields of hippocampus, and CAT, volBrain and BrainSuite automated methods to segment the whole brain using T1 weighted MRI data. Our results showed that all subfields of hippocampus in the Alzheimer's Disease (AD) group were significantly smaller than those of the healthy control (HC) group. The atrophy of all subcomponents of hippocampus were correlated with the MMSE measure. Quite a large portion of cortical and subcortical areas in the brain were also smaller in the AD group as compared to the control group, as evident from CAT and volBrain segmentation results. The shrinkage in these brain areas mostly showed a strong correlation with MMSE measure. BrainSuite failed to discriminate between the two groups. While CAT and volBrain shows a strong correlation, BrainSuite did not show any significant correlation with CAT and volBrain. With the advancement of computational methods, fine-grain analysis of the brain areas is more feasible ${ }^{73-75}$. Earlier methods relied heavily on manual segmentation of the brain areas, which was extremely time demanding and also required a great level of expertise. Therefore, the majority of the analysis was limited to brain areas with more distinct structure, such as the hippocampus. Many semi- and fully-automated segmentation methods have been developed. While these methods have been used more commonly in recent years, the reliability and accuracy of these methods was yet to be fully studied. We used four pipelines of HIPS ${ }^{33}$, volBrain ${ }^{34}$, CAT ${ }^{35,36}$ and BrainSuite ${ }^{37}$. In this study we evaluated their reliability by looking at their ability to discriminate between $\mathrm{AD}$ and $\mathrm{HC}$ groups, whether a correlation existed between them, their correlation with MMSE scores, and comparing their results with past literature. Our results showed strong reliability of HIPS, volBrain and CAT. These methods have been successfully applied to brain images from those with $\mathrm{AD}^{76-79}$.

BrainSuite, however, underperformed greatly. For example, it failed to accurately segment the hippocampus, thalamus and amygdala to show a significant difference between the two groups. While this automatic segmentation method has been used frequently in past research ${ }^{80}$, its application has been mostly limited to the processing of brains with no atrophy ${ }^{81,82}$, as well as detection of gross segments such as tumours ${ }^{83}$. This highlights the importance of validation studies such as ours to gain a greater understanding of the applications and limitations of different methods ${ }^{84-87}$.

The volume of the hippocampus is considered as an important biomarker for AD and has been included in recently proposed research diagnostic criteria ${ }^{88,89}$. It has been shown that the 
hippocampal atrophy estimated on anatomical T1 weighted MRI can help in classifying the different stages of $\mathrm{AD}{ }^{90-92}$. Confirming past literature, our results showed that the hippocampus volume significantly differed between AD and the control group.

Histological studies have shown that lesions are not uniformly distributed within the hippocampus ${ }^{91,93}$. Neuronal loss results in a reduction of the thickness of the layers richer in neuronal bodies, while the loss of synapses results in the reduction of the layers poorer in neuronal bodies ${ }^{94-97}$ and these changes are stage-dependent ${ }^{98,99}$. Our results, however, failed to differentiate the contribution of these subfields in $\mathrm{AD}$; they all showed significant reduction in size, compared to the control group. This effect could be because our AD group consisted of those with later stages of AD. The contribution of different subfields of the hippocampus is more visible in those with mild cognitive impairment ${ }^{100-102}$. Therefore, in future studies it would be informative to include participants with different stages of AD to investigate the contribution of different subfields of the hippocampus in AD.

While the contribution of atrophy in the hippocampus has been widely studied, the role of atrophy in the rest of the brain in $\mathrm{AD}$ is less clear ${ }^{40}$. An important contributing factor is that the boundaries of the hippocampus are easier for human operators or automated algorithms to recognise than other brain areas such as the amygdala, entorhinal cortex or thalamus ${ }^{40}$. Due to methodological advances, however, it is now possible to measure atrophy across the entire cortex with good precision ${ }^{103}$. Our results from CAT and volBrain methods showed strongly significant differences between many brain areas such as the amygdala, thalamus, nucleus accumbens, insula and caudate. These findings are in-line with past literature showing similar differences in these brain areas ${ }^{40,104-106}$.

There is a growing body of literature showing a correlation between cognitive decline and brain atrophy ${ }^{62,107,108}$. For example, it has been shown that basal forebrain changes are correlated with cognitive decline in MCI and AD patients, as measured with recall task and MMSE ${ }^{109,110}$, as well as healthy participants that later progressed to $\mathrm{AD}^{111}$. Atrophy of other brain areas such as lateral and medial parietal cortex, as well as lateral temporal cortex have also been shown to have a correlation with cognitive decline ${ }^{112}$. Our results showed a strong correlation between brain atrophy and cognitive decline as measured by MMSE. All brain areas that were significantly different between the AD and the control group showed a significant correlation with MMSE, except for the caudate (CAT $p=0.001155$, volBrain $p=0.005091$, Bonferroni corrected statistic not significant). While the effect of shrinkage of the caudate in AD is not very clear ${ }^{113}$, there is some evidence that caudate volume has a correlation with MMSE 
measures, although not as strongly as other brain areas such as the thalamus ${ }^{63,114}$. An important consideration is that atrophy in the left caudate has a stronger role in $\mathrm{AD}$, as compared to the right caudate ${ }^{113,115}$. Our analysis combined both the left and right caudate, which may have led to this inconsistency between our results and previous literature.

Although AD commonly presents as an amnestic syndrome, there is significant heterogeneity across individuals ${ }^{116}$, which is accompanied by different atrophy patterns ${ }^{64,117-119}$. For example, while those with more language difficulties might exhibit greater atrophy in temporal or parietal regions ${ }^{120,121}$, those with more visual difficulties might have greater atrophy in posterior cortical regions 122,123. Availability of the automated systems offers many opportunities, such as the ability to analyse a large number of brain images with reasonable time and expertise. This is in particular very appealing, considering the increased number of large datasets such as MIRIAD. Automated systems can go through the collection and aggregate data from a wide range of participants, healthy and patients to gain a greater understanding of $\mathrm{AD}$. This is important considering the heterogeneity of the disease and its $\operatorname{progression}^{124}$.

Another application of automated systems is in clinical settings. By the time of diagnosis, rapid ongoing atrophy is already far advanced 125,126. Early diagnosis of AD can help with deceleration of the progression of the disease ${ }^{21,127,128}$. This is particularly important as there are modifiable factors that can help with brain health ${ }^{129-131}$. Therefore, a massive effort has been devoted to the development of diagnostic methods to enable researchers and clinicians to detect $\mathrm{AD}$ and cases with potential progression to $\mathrm{AD}$, as early as possible ${ }^{132,133}$. For the development of preventive strategies, it is important to predict future brain atrophy, as this may aid in identifying which individuals with normal cognition are more susceptible of progressing to later stages of $\mathrm{AD}{ }^{134-136}$. Automated systems provide additional information to clinicians, enabling them to have a greater understanding of the progression of the atrophy ${ }^{12,137}$. Some of these methods have already received approval from different licensing bodies such as CE (European conformity) and FDA (food and drug administration, USA) approval ${ }^{138}$. These methods, however, come with some limitations such as speed of processing, expensive licences, or requirement of other specialised software. This study is another step to evaluate freely available analytical tools to achieve an ideal analysis pipeline, suitable for researchers and clinicians.

Availability of the reliable automated segmentation methods enables researchers and clinicians to have a greater understanding of the underlying mechanisms and the progression of the AD. 
This will allow them to attempt to prevent or decelerate the progression of the disease more effectively. Future research can look at the rate of atrophy to predict the progression of disease 139-142. This rate can be helpful to have a more informed understanding whether an individual with MCI will later progress to AD or not ${ }^{105,143,144}$. The output of automated segmentation methods can also be used in training of intelligent classification methods such as those using artificial neural networks and support vector machines, which has shown promising results ${ }^{145-}$ 154 .

The purpose of this article was not to identify the superiority of any particular automatic segmentation method over another, but to solely highlight possible limitations and applications of four commonly used segmentation methods. We proposed that CAT, volBrain and HIPS are methods that can robustly operate on brain images with significant atrophy and can be used in research and clinical settings. BrainSuite, however, should be used with caution for brain images with atrophy.

\section{Authors' Contribution}

$\mathrm{JZ}$ and AHJ analysed the data. JZ and AHJ wrote the manuscript. JZ, AHJ and AS revised the manuscript.

\section{References}

1. Scheltens, P. et al. Alzheimer's disease. The Lancet 388, 505-517 (2016).

2. World Health Organization. Fact Sheet-Dementia. (2015).

3. Association, A. 2019 Alzheimer's disease facts and figures. Alzheimer's \& Dementia 15, 321-387 (2019).

4. Nichols, E. et al. Global, regional, and national burden of Alzheimer's disease and other dementias, 1990-2016: a systematic analysis for the Global Burden of Disease Study 2016. The Lancet Neurology 18, 88-106 (2019).

5. Knapp, M. et al. Cost-effectiveness of donepezil and memantine in moderate to severe Alzheimer's disease (the DOMINO-AD trial). International Journal of Geriatric Psychiatry 32, 1205-1216 (2017). 
6. Montine, T. J. et al. National institute on aging-Alzheimer's association guidelines for the neuropathologic assessment of Alzheimer's disease: A practical approach. Acta Neuropathologica 123, 1-11 (2012).

7. Duyckaerts, C., Delatour, B. \& Potier, M. C. Classification and basic pathology of Alzheimer disease. Acta Neuropathologica 118, 5-36 (2009).

8. Edwards, F. A. A Unifying Hypothesis for Alzheimer's Disease: From Plaques to Neurodegeneration. Trends in Neurosciences 42, 310-322 (2019).

9. McConathy, J. \& Sheline, Y. I. Imaging biomarkers associated with cognitive decline: A review. Biological Psychiatry 77, 685-692 (2015).

10. Tabatabaei-Jafari, H., Shaw, M. E. \& Cherbuin, N. Cerebral atrophy in mild cognitive impairment: A systematic review with meta-analysis. Alzheimer's and Dementia: Diagnosis, Assessment and Disease Monitoring 1, 487-504 (2015).

11. Grundman, M. et al. Mild Cognitive Impairment Can Be Distinguished from Alzheimer Disease and Normal Aging for Clinical Trials. Archives of Neurology 61, 59-66 (2004).

12. Frisoni, G. B., Fox, N. C., Jack, C. R., Scheltens, P. \& Thompson, P. M. The clinical use of structural MRI in Alzheimer disease. Nature Reviews Neurology 6, 67-77 (2010).

13. Mueller, S. G. et al. Hippocampal atrophy patterns in mild cognitive impairment and alzheimer's disease. Human Brain Mapping 31, 1339-1347 (2010).

14. Kerchner, G. A. et al. Hippocampal CA1 apical neuropil atrophy in mild Alzheimer disease visualized with 7-T MRI. Neurology 75, 1381-1387 (2010).

15. Yassa, M. A. et al. High-resolution structural and functional MRI of hippocampal CA3 and dentate gyrus in patients with amnestic Mild Cognitive Impairment. NeuroImage 51, 1242-1252 (2010).

16. Adachi, M. et al. Morphology of the inner structure of the hippocampal formation in Alzheimer disease. American Journal of Neuroradiology 24, 1575-1581 (2003).

17. McRae-McKee, K. et al. Combining hippocampal volume metrics to better understand Alzheimer's disease progression in at-risk individuals. Scientific Reports $\mathbf{9}$, $1-9$ (2019). 
18. de Flores, R., la Joie, R. \& Chételat, G. Structural imaging of hippocampal subfields in healthy aging and Alzheimer's disease. Neuroscience 309, 29-50 (2015).

19. Nobis, L. et al. Hippocampal volume across age: Nomograms derived from over 19,700 people in UK Biobank. NeuroImage: Clinical 23, 101904 (2019).

20. Mueller, S. G. \& Weiner, M. W. Selective effect of age, Apo e4, and Alzheimer's disease on hippocampal subfields. Hippocampus 19, 558-564 (2009).

21. Nestor, P. J., Scheltens, P. \& Hodges, J. R. Advances in the early detection of Alzheimer's disease. Nature Medicine 10, S34-S41 (2004).

22. Wisse, L. E. M. et al. Hippocampal subfield volumes at 7T in early Alzheimer's disease and normal aging. Neurobiology of Aging 35, 2039-2045 (2014).

23. Braak, H. \& Braak, E. Alzheimer's disease affects limbic nuclei of the thalamus. Acta Neuropathologica 81, 261-268 (1991).

24. Bobinski, M. et al. Relationships between Regional Neuronal Loss and Neurofibrillary Changes in the Hippocampal Formation and Duration and Severity of Alzheimer Disease. Journal of Neuropathology and Experimental Neurology 56, 414 420 (1997).

25. Schönheit, B., Zarski, R. \& Ohm, T. G. Spatial and temporal relationships between plaques and tangles in Alzheimer-pathology. Neurobiology of Aging 25, 697-711 (2004).

26. Li, F., Ball, S., Katz, B. \& Smith, A. Case report of syncope during a transcranial direct current stimulation experiment in a healthy adult participant. Brain Stimulation 0, 1201-1202 (2018).

27. la Joie, R. et al. Hippocampal subfield volumetry in mild cognitive impairment, Alzheimer's disease and semantic dementia. NeuroImage: Clinical 3, 155-162 (2013).

28. Litjens, G. et al. A survey on deep learning in medical image analysis. Medical Image Analysis 42, 60-88 (2017).

29. Han, X. et al. Reliability of MRI-derived measurements of human cerebral cortical thickness: The effects of field strength, scanner upgrade and manufacturer.

NeuroImage 32, 180-194 (2006). 
30. Ambarki, K., Wåhlin, A., Birgander, R., Eklund, A. \& Malm, J. MR imaging of brain volumes: Evaluation of a fully automatic software. American Journal of Neuroradiology 32, 408-412 (2011).

31. Kerchner, G. A. et al. Shared vulnerability of two synaptically-connected medial temporal lobe areas to age and cognitive decline: A seven tesla magnetic resonance imaging study. Journal of Neuroscience 33, 16666-16672 (2013).

32. Yushkevich, P. A. et al. Automated volumetry and regional thickness analysis of hippocampal subfields and medial temporal cortical structures in mild cognitive impairment. Human Brain Mapping 36, 258-287 (2015).

33. Romero, J. E., Coupé, P. \& Manjón, J. v. HIPS: A new hippocampus subfield segmentation method. NeuroImage 163, 286-295 (2017).

34. Manjón, J. v. \& Coupé, P. Volbrain: An online MRI brain volumetry system. Frontiers in Neuroinformatics 10, 1-14 (2016).

35. Tzourio-Mazoyer, N. et al. Automated Anatomical Labeling of Activations in SPM Using a Macroscopic Anatomical Parcellation of the MNI MRI Single-Subject Brain. NeuroImage 15, 273-289 (2002).

36. Rolls, E. T., Huang, C.-C., Lin, C.-P., Feng, J. \& Joliot, M. Automated anatomical labelling atlas 3. NeuroImage 206, 116189 (2020).

37. Shattuck, D. W. \& Leahy, R. M. BrainSuite: An automated cortical surface identification tool. Medical Image Analysis 6, 129-142 (2002).

38. Shattuck, D. W. et al. Semi-automated method for delineation of landmarks on models of the cerebral cortex. Journal of Neuroscience Methods 178, 385-392 (2009).

39. Yushkevich, P. A. et al. Nearly automatic segmentation of hippocampal subfields in in vivo focal T2-weighted MRI. Neurolmage 53, 1208-1224 (2010).

40. Pini, L. et al. Brain atrophy in Alzheimer's Disease and aging. Ageing Research Reviews 30, 25-48 (2016).

41. Bigler, E. D. et al. FreeSurfer 5.3 versus 6.0: are volumes comparable? A Chronic Effects of Neurotrauma Consortium study. Brain Imaging and Behavior (2018) doi:10.1007/s11682-018-9994-x. 
42. Kook Lim, H. et al. Automated hippocampal subfields segmentation in late life depression. Journal of Affective Disorders 143, 253-256 (2012).

43. Hanseeuw, B. J. et al. Mild cognitive impairment: Differential atrophy in the hippocampal subfields. American Journal of Neuroradiology 32, 1658-1661 (2011).

44. Li, Y. di, Dong, H. B., Xie, G. M. \& Zhang, L. J. Discriminative analysis of mild alzheimer's disease and normal aging using volume of hippocampal subfields and hippocampal mean diffusivity: An in vivo magnetic resonance imaging study. American Journal of Alzheimer's Disease and other Dementias 28, 627-633 (2013).

45. Khan, U. A. et al. Molecular drivers and cortical spread of lateral entorhinal cortex dysfunction in preclinical Alzheimer's disease. Nature Neuroscience 17, 304-311 (2014).

46. Wisse, L. E. M., Biessels, G. J. \& Geerlings, M. I. A Critical Appraisal of the Hippocampal Subfield Segmentation Package in FreeSurfer. Frontiers in Aging Neuroscience 6, 127-134 (2014).

47. Cover, K. S., van Schijndel, R. A., Bosco, P., Damangir, S. \& Redolfi, A. Can measuring hippocampal atrophy with a fully automatic method be substantially less noisy than manual segmentation over both 1 and 3 years? Psychiatry Research Neuroimaging 280, 39-47 (2018).

48. Marizzoni, M. et al. Longitudinal reproducibility of automatically segmented hippocampal subfields: A multisite European 3T study on healthy elderly. Human Brain Mapping 36, 3516-3527 (2015).

49. Bender, A. R. et al. Optimization and validation of automated hippocampal subfield segmentation across the lifespan. Human Brain Mapping 39, 916-931 (2018).

50. Pantazis, D. et al. Comparison of landmark-based and automatic methods for cortical surface registration. NeuroImage 49, 2479-2493 (2010).

51. Malone, I. B. et al. MIRIAD-Public release of a multiple time point Alzheimer's MR imaging dataset. NeuroImage 70, 33-36 (2013).

52. Iglesias, J. E. et al. Bayesian longitudinal segmentation of hippocampal substructures in brain MRI using subject-specific atlases. NeuroImage 141, 542-555 (2016). 
53. Cash, D. M. et al. Assessing atrophy measurement techniques in dementia: Results from the MIRIAD atrophy challenge. NeuroImage 123, 149-164 (2015).

54. Platero, C., Lin, L. \& Tobar, M. C. Longitudinal Neuroimaging Hippocampal Markers for Diagnosing Alzheimer's Disease. Neuroinformatics 17, 43-61 (2019).

55. Giraldo, D. L., García-Arteaga, J. D., Cárdenas-Robledo, S. \& Romero, E. Characterization of brain anatomical patterns by comparing region intensity distributions: Applications to the description of Alzheimer's disease. Brain and Behavior 8, 1-11 (2018).

56. Ardekani, B. A., Convit, A. \& Bachman, A. H. Analysis of the MIRIAD Data Shows Sex Differences in Hippocampal Atrophy Progression. Journal of Alzheimer's Disease 50, 847-857 (2016).

57. Carrillo, M. C., Bain, L. J., Frisoni, G. B. \& Weiner, M. W. Worldwide Alzheimer's disease neuroimaging initiative. Alzheimer's and Dementia 8, 337-342 (2012).

58. van Horn, J. D. \& Toga, A. W. Multisite neuroimaging trials. Current Opinion in Neurology 22, 370-378 (2009).

59. Schneider, L. S. \& Goldberg, T. E. Composite cognitive and functional measures for early stage Alzheimer's disease trials. Alzheimer's \& Dementia: Diagnosis, Assessment \& Disease Monitoring 12, 1-9 (2020).

60. Dicks, E. et al. Modeling grey matter atrophy as a function of time, aging or cognitive decline show different anatomical patterns in Alzheimer's disease. NeuroImage: Clinical 22, 101786 (2019).

61. Folstein, M. F., Folstein, S. E. \& McHugh, P. R. "Mini-mental state": a practical method for grading the cognitive state of patients for the clinician. Journal of psychiatric research 12, 189-198 (1975).

62. Nelson, P. T. et al. Correlation of Alzheimer Disease Neuropathologic Changes With Cognitive Status: A Review of the Literature. Journal of Neuropathology \& Experimental Neurology 71, 362-381 (2012).

63. Ferrarini, L. et al. MMSE scores correlate with local ventricular enlargement in the spectrum from cognitively normal to Alzheimer disease. NeuroImage 39, 1832-1838 (2008). 
64. Risacher, S. L. et al. Alzheimer disease brain atrophy subtypes are associated with cognition and rate of decline. Neurology 89, 2176-2186 (2017).

65. Tustison, N. J. et al. N4ITK: Improved N3 bias correction. IEEE Transactions on Medical Imaging 29, 1310-1320 (2010).

66. Manjón, J. v., Coupé, P., Martí-Bonmatí, L., Collins, D. L. \& Robles, M. Adaptive non-local means denoising of MR images with spatially varying noise levels. Journal of Magnetic Resonance Imaging 31, 192-203 (2010).

67. Coupé, P. et al. Patch-based segmentation using expert priors: Application to hippocampus and ventricle segmentation. NeuroImage 54, 940-954 (2011).

68. Romero, J. E., Manjón, J. v., Tohka, J., Coupé, P. \& Robles, M. NABS: Non-local automatic brain hemisphere segmentation. Magnetic Resonance Imaging 33, 474-484 (2015).

69. Ashburner, J. \& Friston, K. J. Unified segmentation. NeuroImage 26, 839-851 (2005).

70. Gaser, C., \& Dahnke, R. CAT-A Computational Anatomy Toolbox for the Analysis of Structural MRI Data. Human Brain Mapping (2016).

71. Dahnke, R. \& Gaser, C. Brain structural trajectories over the adult lifespan. Human Brain Mapping (2017).

72. Manjón, J. v., Coupé, P., Martí-Bonmatí, L., Collins, D. L. \& Robles, M. Adaptive non-local means denoising of MR images with spatially varying noise levels. Journal of Magnetic Resonance Imaging 31, 192-203 (2010).

73. Brewer, J. B., Magda, S., Airriess, C. \& Smith, M. E. Fully-automated quantification of regional brain volumes for improved detection of focal atrophy in Alzheimer disease. American Journal of Neuroradiology 30, 578-580 (2009).

74. Hata, K. et al. Automated Volumetry of Medial Temporal Lobe Subregions in Mild Cognitive Impairment and Alzheimer Disease. Alzheimer Disease and Associated Disorders 33, 206-211 (2019).

75. Fischl, B. et al. Whole brain segmentation: Automated labeling of neuroanatomical structures in the human brain. Neuron 33, 341-355 (2002). 
76. Haeger, A. et al. Effect of a multicomponent exercise intervention on brain metabolism: A randomized controlled trial on Alzheimer's pathology (DementiaMOVE). Alzheimer's \& Dementia: Translational Research \& Clinical Interventions 6 , $1-9(2020)$.

77. Coupé, P., Manjón, J. V., Lanuza, E. \& Catheline, G. Lifespan Changes of the Human Brain In Alzheimer's Disease. Scientific Reports 9, 1-12 (2019).

78. Planche, V. et al. Evolution of brain atrophy subtypes during aging predicts longterm cognitive decline and future Alzheimer's clinical syndrome. Neurobiology of Aging 79, 22-29 (2019).

79. Goubran, M. et al. Hippocampal segmentation for brains with extensive atrophy using three-dimensional convolutional neural networks. Human Brain Mapping 41, 291-308 (2020).

80. Wang, Z. I. et al. Automated MRI volumetric analysis in patients with rasmussen syndrome. American Journal of Neuroradiology 37, 2348-2355 (2016).

81. Skjøth-Rasmussen, J., Jespersen, B. \& Brennum, J. The use of Brainsuite iCT for frame-based stereotactic procedures. Acta Neurochirurgica 157, 1437-1440 (2015).

82. Ou, Y. et al. Field of View Normalization in Multi-Site Brain MRI. Neuroinformatics 16, 431-444 (2018).

83. D'Andrea, G. et al. Intraoperative DTI and brain mapping for surgery of neoplasm of the motor cortex and the corticospinal tract: Our protocol and series in BrainSUITE. Neurosurgical Review 35, 401-412 (2012).

84. Mikhael, S. S. \& Pernet, C. A controlled comparison of thickness, volume and surface areas from multiple cortical parcellation packages. BMC Bioinformatics 20, 1$12(2019)$.

85. Mikhael, S., Hoogendoorn, C., Valdes-Hernandez, M. \& Pernet, C. A critical analysis of neuroanatomical software protocols reveals clinically relevant differences in parcellation schemes. NeuroImage 170, 348-364 (2018).

86. Carass, A. et al. Comparing fully automated state-of-the-art cerebellum parcellation from magnetic resonance images. NeuroImage 183, 150-172 (2018). 
87. Schwarz, C. G. et al. A large-scale comparison of cortical thickness and volume methods for measuring Alzheimer's disease severity. NeuroImage: Clinical 11, 802812 (2016).

88. Jack, C. R. et al. Introduction to the recommendations from the National Institute on Aging-Alzheimer's Association workgroups on diagnostic guidelines for Alzheimer's disease. Alzheimer's and Dementia 7, 257-262 (2011).

89. Albert, M. S. et al. The diagnosis of mild cognitive impairment due to Alzheimer's disease: Recommendations from the National Institute on Aging-Alzheimer's Association workgroups on diagnostic guidelines for Alzheimer's disease. Alzheimer's and Dementia 7, 270-279 (2011).

90. Anderson, V. M. et al. Gray matter atrophy rate as a marker of disease progression in AD. Neurobiology of Aging 33, 1194-1202 (2012).

91. Boutet, C. et al. Detection of volume loss in hippocampal layers in Alzheimer's disease using 7 T MRI: A feasibility study. NeuroImage: Clinical 5, 341-348 (2014).

92. Adler, D. H. et al. Characterizing the human hippocampus in aging and Alzheimer's disease using a computational atlas derived from ex vivo MRI and histology. Proceedings of the National Academy of Sciences of the United States of America 115, 4252-4257 (2018).

93. Kwak, K., Niethammer, M., Giovanello, K. S., Styner, M. \& Dayan, E. The contribution of hippocampal subfields to the progression of neurodegeneration. bioRxiv (2020) doi:10.1101/2020.05.06.081034.

94. Lace, G. et al. Hippocampal tau pathology is related to neuroanatomical connections: An ageing population-based study. Brain 132, 1324-1334 (2009).

95. Delgado-González, J. C. et al. Neuronal volume of the hippocampal regions in ageing. Journal of Anatomy 1-10 (2020) doi:10.1111/joa.13189.

96. Parker, T. D. et al. Differences in hippocampal subfield volume are seen in phenotypic variants of early onset Alzheimer's disease. NeuroImage: Clinical 21, 101632 (2019).

97. Carlesimo, G. A. et al. Atrophy of presubiculum and subiculum is the earliest hippocampal anatomical marker of Alzheimer's disease. Alzheimer's and Dementia: Diagnosis, Assessment and Disease Monitoring 1, 24-32 (2015). 
98. Rössler, M., Zarski, R., Bohl, J. \& Ohm, T. G. Stage-dependent and sector-specific neuronal loss in hippocampus during alzheimer's disease. Acta Neuropathologica 103, 363-369 (2002).

99. Braak, E. \& Braak, H. Alzheimer's disease: Transiently developing dendritic changes in pyramidal cells of sector CA1 of the Ammon's horn. Acta Neuropathologica 93, 323-325 (1997).

100. Su, L. et al. Hippocampal Stratum Radiatum, Lacunosum, and Moleculare Sparing in Mild Cognitive Impairment. Journal of Alzheimer's Disease 61, 415-424 (2018).

101. Dounavi, M. E. et al. Volumetric alterations in the hippocampal subfields of subjects at increased risk of dementia. Neurobiology of Aging 91, 36-44 (2020).

102. Zhao, W. et al. Trajectories of the hippocampal subfields atrophy in the alzheimer's disease: A structural imaging study. Frontiers in Neuroinformatics 13, 1-9 (2019).

103. Khlif, M. S. et al. A comparison of automated segmentation and manual tracing in estimating hippocampal volume in ischemic stroke and healthy control participants. NeuroImage: Clinical 21, (2019).

104. Tang, X., Holland, D., Dale, A. M., Younes, L. \& Miller, M. I. Shape abnormalities of subcortical and ventricular structures in mild cognitive impairment and Alzheimer's disease: Detecting, quantifying, and predicting. Human Brain Mapping 35, 3701-3725 (2014).

105. Yi, H. A. et al. Relation between subcortical grey matter atrophy and conversion from mild cognitive impairment to Alzheimer's disease. Journal of Neurology, Neurosurgery and Psychiatry 87, 425-432 (2016).

106. Tullo, S. et al. MR-based age-related effects on the striatum, globus pallidus, and thalamus in healthy individuals across the adult lifespan. Human Brain Mapping 40, 5269-5288 (2019).

107. Mufson, E. J. et al. Mild cognitive impairment: Pathology and mechanisms. Acta Neuropathologica 123, 13-30 (2012).

108. Dicks, E., van der Flier, W. M., Scheltens, P., Barkhof, F. \& Tijms, B. M. Singlesubject grey matter networks predict future cortical atrophy in preclinical Alzheimer's disease. Neurobiology of Aging (2020) doi:10.1016/j.neurobiolaging.2020.05.008. 
109. Grothe, M. et al. Reduction of basal forebrain cholinergic system parallels cognitive impairment in patients at high risk of developing alzheimer's disease. Cerebral Cortex 20, 1685-1695 (2010).

110. Muth, K. et al. Mild Cognitive Impairment in the Elderly is Associated with Volume Loss of the Cholinergic Basal Forebrain Region. Biological Psychiatry 67, 588-591 (2010).

111. Grothe, M., Heinsen, H. \& Teipel, S. Longitudinal measures of cholinergic forebrain atrophy in the transition from healthy aging to Alzheimer's disease. Neurobiology of Aging 34, 1210-1220 (2013).

112. Ossenkoppele, R. et al. Associations between tau, A $\beta$, and cortical thickness with cognition in Alzheimer disease. Neurology 92, e601-e612 (2019).

113. Barber, R., McKeith, I., Ballard, C. \& O'Brien, J. Volumetric MRI study of the caudate nucleus in patients with dementia with Lewy bodies, Alzheimer's disease, and vascular dementia. Journal of Neurology Neurosurgery and Psychiatry 72, 406-407 (2002).

114. Yan, T. et al. Early-Stage Identification and Pathological Development of Alzheimer's Disease Using Multimodal MRI. Journal of Alzheimer's Disease 68 , 1013-1027 (2019).

115. Botzung, A., Philippi, N., Noblet, V., Loureiro De Sousa, P. \& Blanc, F. Pay attention to the basal ganglia: A volumetric study in early dementia with Lewy bodies. Alzheimer's Research and Therapy 11, 1-9 (2019).

116. Sun, N., Mormino, E. C., Chen, J., Sabuncu, M. R. \& Yeo, B. T. T. Multi-modal latent factor exploration of atrophy, cognitive and tau heterogeneity in Alzheimer's disease. NeuroImage 201, (2019).

117. Young, A. L. et al. Uncovering the heterogeneity and temporal complexity of neurodegenerative diseases with Subtype and Stage Inference. Nature Communications 9, 1-16 (2018).

118. Noh, Y. et al. Anatomical heterogeneity of Alzheimer disease Based on cortical thickness on MRIs. Neurology 83, 1936-1944 (2014). 
119. Ferreira, D. et al. Distinct subtypes of Alzheimer's disease based on patterns of brain atrophy: Longitudinal trajectories and clinical applications. Scientific Reports 7, 1-13 (2017).

120. Gorno-Tempini, M. L. et al. Classification of primary progressive aphasia and its variants. Neurology 76, 1006-1014 (2011).

121. Phillips, J. S. et al. Tau PET imaging predicts cognition in atypical variants of Alzheimer's disease. Human Brain Mapping 39, 691-708 (2018).

122. Ossenkoppele, R. et al. The behavioural/dysexecutive variant of Alzheimer's disease: Clinical, neuroimaging and pathological features. Brain 138, 2732-2749 (2015).

123. Ossenkoppele, R. et al. Tau PET patterns mirror clinical and neuroanatomical variability in Alzheimer's disease. Brain 139, 1551-1567 (2016).

124. Arbabshirani, M. R., Plis, S., Sui, J. \& Calhoun, V. D. Single subject prediction of brain disorders in neuroimaging: Promises and pitfalls. NeuroImage 145, 137-165 (2017).

125. Spires-Jones, T. L. \& Hyman, B. T. The Intersection of Amyloid Beta and Tau at Synapses in Alzheimer's Disease. Neuron 82, 756-771 (2014).

126. McKhann, G. M. et al. The diagnosis of dementia due to Alzheimer's disease: Recommendations from the National Institute on Aging-Alzheimer's Association workgroups on diagnostic guidelines for Alzheimer's disease. Alzheimer's and Dementia 7, 263-269 (2011).

127. Sørensen, L. et al. Early detection of Alzheimer's disease using MRI hippocampal texture. Human Brain Mapping 37, 1148-1161 (2016).

128. McEvoy, L. K. \& Brewer, J. B. Quantitative structural MRI for early detection of Alzheimers disease. Expert Review of Neurotherapeutics 10, 1675-1688 (2010).

129. Fotuhi, M., Do, D. \& Jack, C. Modifiable factors that alter the size of the hippocampus with ageing. Nature Reviews Neurology 8, 189-202 (2012).

130. Erickson, K. I. et al. Exercise training increases size of hippocampus and improves memory. Proceedings of the National Academy of Sciences 108, 3017-3022 (2011). 
131. Varma, V. R., Chuang, Y. F., Harris, G. C., Tan, E. J. \& Carlson, M. C. Lowintensity daily walking activity is associated with hippocampal volume in older adults. Hippocampus 25, 605-615 (2015).

132. Liang, Y., Ryan, N. S., Schott, J. M. \& Fox, N. C. Imaging the onset and progression of Alzheimer's disease: Implications for prevention trials. Journal of Alzheimer's Disease 33, (2013).

133. Coupé, P., Eskildsen, S. F., Manjón, J. v., Fonov, V. S. \& Collins, D. L. Simultaneous segmentation and grading of anatomical structures for patient's classification: Application to Alzheimer's disease. NeuroImage 59, 3736-3747 (2012).

134. Sperling, R. A. et al. Toward defining the preclinical stages of Alzheimer's disease: Recommendations from the National Institute on Aging-Alzheimer's Association workgroups on diagnostic guidelines for Alzheimer's disease. Alzheimer's and Dementia 7, 280-292 (2011).

135. Veitch, D. P. et al. Understanding disease progression and improving Alzheimer's disease clinical trials: Recent highlights from the Alzheimer's Disease Neuroimaging Initiative. Alzheimer's and Dementia 15, 106-152 (2019).

136. Vanhoenacker, A. S., Sneyers, B., de Keyzer, F., Heye, S. \& Demaerel, P. Evaluation and clinical correlation of practical cut-offs for visual rating scales of atrophy: normal aging versus mild cognitive impairment and Alzheimer's disease. Acta Neurologica Belgica 117, 661-669 (2017).

137. Shaw, T., York, A., Ziaei, M., Barth, M. \& Bollmann, S. Longitudinal Automatic Segmentation of Hippocampal Subfields (LASHiS) using Multi-Contrast MRI. NeuroImage 116798 (2020) doi:10.1016/j.neuroimage.2020.116798.

138. Scarpazza, C. et al. Translating research findings into clinical practice: a systematic and critical review of neuroimaging-based clinical tools for brain disorders.

\section{Translational Psychiatry 10, (2020).}

139. Henneman, W. J. P. et al. Hippocampal atrophy rates in Alzheimer disease: Added value over whole brain volume measures. Neurology 72, 999-1007 (2009).

140. Leung, K. K. et al. Automated cross-sectional and longitudinal hippocampal volume measurement in mild cognitive impairment and Alzheimer's disease. NeuroImage 51, 1345-1359 (2010). 
141. Frankó, E. \& Joly, O. Evaluating Alzheimer's Disease Progression Using Rate of Regional Hippocampal Atrophy. PLoS ONE 8, (2013).

142. Mak, E. et al. Longitudinal assessment of global and regional atrophy rates in Alzheimer's disease and dementia with Lewy bodies. NeuroImage: Clinical 7, 456$462(2015)$.

143. Eustache, P., Nemmi, F., Saint-Aubert, L., Pariente, J. \& Péran, P. Multimodal Magnetic Resonance Imaging in Alzheimer's Disease Patients at Prodromal Stage. Journal of Alzheimer's Disease 50, 1035-1050 (2016).

144. Pagani, M. et al. Predicting the transition from normal aging to Alzheimer's disease: A statistical mechanistic evaluation of FDG-PET data. NeuroImage 141, 282-290 (2016).

145. Qiu, S. et al. Development and validation of an interpretable deep learning framework for Alzheimer's disease classification. Brain 1-14 (2020) doi:10.1093/brain/awaa137.

146. Zhao, W. et al. Automated Brain MRI Volumetry Differentiates Early Stages of Alzheimer's Disease From Normal Aging. Journal of Geriatric Psychiatry and Neurology 32, 354-364 (2019).

147. Li, H., Habes, M., Wolk, D. A. \& Fan, Y. A deep learning model for early prediction of Alzheimer's disease dementia based on hippocampal magnetic resonance imaging data. Alzheimer's and Dementia 15, 1059-1070 (2019).

148. Bachli, M. B. et al. Evaluating the reliability of neurocognitive biomarkers of neurodegenerative diseases across countries: A machine learning approach. NeuroImage 208, (2020).

149. Bouts, M. J. R. J. et al. Detection of mild cognitive impairment in a communitydwelling population using quantitative, multiparametric MRI-based classification. Human Brain Mapping 40, 2711-2722 (2019).

150. Forouzannezhad, P. et al. A Gaussian-based model for early detection of mild cognitive impairment using multimodal neuroimaging. Journal of Neuroscience Methods 333, 108544 (2020). 
151. Khan, R. U., Tanveer, M. \& Pachori, R. B. A novel method for the classification of Alzheimer's disease from normal controls using magnetic resonance imaging. Expert Systems 1-22 (2020) doi:10.1111/exsy.12566.

152. Oh, K., Chung, Y. C., Kim, K. W., Kim, W. S. \& Oh, I. S. Classification and Visualization of Alzheimer's Disease using Volumetric Convolutional Neural Network and Transfer Learning. Scientific Reports 9, 1-16 (2019).

153. Dukart, J. et al. Meta-analysis based SVM classification enables accurate detection of Alzheimer's disease across different clinical centers using FDG-PET and MRI.

Psychiatry Research - Neuroimaging 212, 230-236 (2013).

154. Lecun, Y., Bengio, Y. \& Hinton, G. Deep learning. Nature 521, 436-444 (2015). 

made available under aCC-BY-NC-ND 4.0 International license.

Table 1. Comparison between Alzheimer's disease (AD) and healthy controls (HC).

\begin{tabular}{llll}
\hline Measure & Alzheimer's Disease & Healthy Control & $\boldsymbol{p}$ \\
\hline $\mathrm{n}$ & 22 & 22 & - \\
Female (n [\%]) & $11[50]$ & $11[50]$ & - \\
Age (mean [SD]) & $70.64[5.55]$ & $70.61[7.54]$ & 0.916 \\
MMSE (mean [SD]; min-max) & $17.272[5.461] ; 5-26$ & $29.087[1.083] ; 27-30$ & $<0.001$ \\
\hline
\end{tabular}


Table 2. Summary of the independent-sample t-tests comparing volumetric data between the participants with Alzheimer's disease and healthy controls and the correlation of the data with MMSE scores using CAT method.

\begin{tabular}{|c|c|c|c|c|c|}
\hline \multirow[b]{2}{*}{ Brain Area } & \multicolumn{3}{|c|}{ Comparison between groups } & \multicolumn{2}{|c|}{ Correlation with MMSE } \\
\hline & $t$ & $p \uparrow$ & $d$ & $r^{2}$ & $p$ \\
\hline Fusiform Gyrus & 10.793 & $<0.000001^{*}$ & 3.413 & 0.723 & $<0.000001^{*}$ \\
\hline Parahippocampus Gyrus & 9.936 & $<0.000001^{*}$ & 3.142 & 0.536 & $<0.000001^{*}$ \\
\hline Hippocampus & 9.753 & $<0.000001^{*}$ & 3.084 & 0.460 & $0.000001^{*}$ \\
\hline Entorhinal Cortex & 9.717 & $<0.000001^{*}$ & 3.073 & 0.476 & $<0.000001^{*}$ \\
\hline Amygdala & 9.043 & $<0.000001^{*}$ & 2.860 & 0.445 & $0.000001^{*}$ \\
\hline Inferior Temporal Gyrus & 8.939 & $<0.000001^{*}$ & 2.827 & 0.653 & $<0.000001^{*}$ \\
\hline Middle Temporal Gyrus & 7.632 & $<0.000001^{*}$ & 2.413 & 0.619 & $<0.000001^{*}$ \\
\hline Temporal Pole & 7.185 & $<0.000001^{*}$ & 2.272 & 0.491 & $<0.000001^{*}$ \\
\hline Basal Forebrain & 6.658 & $<0.000001^{*}$ & 2.105 & 0.453 & $0.000001^{*}$ \\
\hline Thalamus & 6.344 & $<0.000001^{*}$ & 2.006 & 0.471 & $0.000001^{*}$ \\
\hline Angular Gyrus & 5.808 & $0.000001^{*}$ & 1.837 & 0.507 & $<0.000001^{*}$ \\
\hline Accumbens & 5.275 & $0.000005^{*}$ & 1.668 & 0.289 & $0.000236^{*}$ \\
\hline Inferior Occipital Gyrus & 5.228 & $0.000006^{*}$ & 1.653 & 0.527 & $<0.000001^{*}$ \\
\hline Superior Temporal Gyrus & 5.186 & $0.000007^{*}$ & 1.640 & 0.513 & $<0.000001^{*}$ \\
\hline Supramarginal Gyrus & 5.101 & $0.000009^{*}$ & 1.613 & 0.498 & $<0.000001^{*}$ \\
\hline Anterior Insula & 4.955 & $0.000014^{*}$ & 1.567 & 0.346 & $0.000043^{*}$ \\
\hline Occipital Fusiform Gyrus & 4.519 & $0.000054^{*}$ & 1.429 & 0.472 & $0.000001^{*}$ \\
\hline Middle Occipital Gyrus & 4.515 & $0.000055^{*}$ & 1.428 & 0.417 & $0.000004^{*}$ \\
\hline Posterior Insula & 4.447 & $0.000068^{*}$ & 1.406 & 0.338 & $0.000054^{*}$ \\
\hline Planum Polare & 4.232 & $0.000131^{*}$ & 1.338 & 0.349 & $0.000038^{*}$ \\
\hline Anterior Cingulate Gyrus & 4.225 & $0.000134^{*}$ & 1.336 & 0.362 & $0.000025^{*}$ \\
\hline Superior Parietal Lobule & 4.131 & $0.000179^{*}$ & 1.306 & 0.423 & $0.000003^{*}$ \\
\hline Caudate & 3.959 & $0.000301^{*}$ & 1.252 & 0.234 & 0.001155 \\
\hline Subcallosal Area & 3.847 & $0.000420^{*}$ & 1.217 & 0.299 & $0.000180^{*}$ \\
\hline Middle Frontal Gyrus & 3.796 & $0.000490^{*}$ & 1.200 & 0.328 & $0.000073^{*}$ \\
\hline Medial Orbital Gyrus & 3.739 & $0.000579^{*}$ & 1.182 & 0.278 & $0.000331^{*}$ \\
\hline Inferior Frontal Gyrus & 3.691 & $0.000666^{*}$ & 1.167 & 0.373 & $0.000017^{*}$ \\
\hline Precuneus & 3.633 & $0.000788^{*}$ & 1.149 & 0.349 & $0.000038^{*}$ \\
\hline Superior Medial Frontal Gyrus & 3.543 & 0.001023 & 1.120 & 0.312 & $0.000120^{*}$ \\
\hline Putamen & 3.525 & 0.001077 & 1.115 & 0.203 & 0.002822 \\
\hline Temporal & 3.489 & 0.001195 & 1.103 & 0.340 & $0.000051^{*}$ \\
\hline
\end{tabular}




\begin{tabular}{|c|c|c|c|c|c|}
\hline Anterior Orbital Gyrus & 3.413 & 0.001484 & 1.079 & 0.244 & 0.000894 \\
\hline Posterior Orbital Gyrus & 3.272 & 0.002203 & 1.035 & 0.255 & $0.000643^{*}$ \\
\hline Lingual Gyrus & 3.263 & 0.002262 & 1.032 & 0.304 & $0.000158^{*}$ \\
\hline Posterior Cingulate Gyrus & 3.226 & 0.002506 & 1.020 & 0.314 & $0.000117^{*}$ \\
\hline Central Operculum & 3.116 & 0.003388 & 0.985 & 0.372 & $0.000018^{*}$ \\
\hline Frontal Operculum & 2.999 & 0.004638 & 0.948 & 0.265 & $0.000479^{*}$ \\
\hline Supplementary Motor Cortex & 2.969 & 0.005032 & 0.939 & 0.310 & $0.000128^{*}$ \\
\hline Exterior Cerebellum & 2.934 & 0.005513 & 0.928 & 0.163 & 0.007986 \\
\hline Superior Frontal Gyrus & 2.870 & 0.006523 & 0.908 & 0.199 & 0.003066 \\
\hline Parietal Operculum & 2.864 & 0.006637 & 0.906 & 0.320 & $0.000095^{*}$ \\
\hline Middle Cingulate Gyrus & 2.734 & 0.009272 & 0.865 & 0.269 & $0.000432^{*}$ \\
\hline Gyrus Rectus & 2.660 & 0.011190 & 0.841 & 0.143 & 0.013603 \\
\hline Optic Chiasm & 2.531 & 0.015401 & 0.800 & 0.066 & 0.101456 \\
\hline Lateral Orbital Gyrus & 2.427 & 0.019841 & 0.767 & 0.158 & 0.009194 \\
\hline Temporal Transverse Gyrus & 2.345 & 0.024056 & 0.742 & 0.189 & 0.003960 \\
\hline Superior Occipital Gyrus & 2.290 & 0.027347 & 0.724 & 0.245 & 0.000849 \\
\hline Medial Precentral Gyrus & 2.250 & 0.030015 & 0.712 & 0.196 & 0.003321 \\
\hline Cuneus & 2.091 & 0.042968 & 0.661 & 0.187 & 0.004135 \\
\hline Inferior Frontal Orbital Gyrus & 2.063 & 0.045679 & 0.652 & 0.123 & 0.022887 \\
\hline Postcentral Gyrus & 1.687 & 0.099402 & 0.533 & 0.142 & 0.013747 \\
\hline Frontal Pole & 1.603 & 0.116854 & 0.507 & 0.067 & 0.098513 \\
\hline Occipital Pole & 1.596 & 0.118311 & 0.505 & 0.127 & 0.020199 \\
\hline Inferior Frontal Angular Gyrus & 1.518 & 0.136864 & 0.480 & 0.134 & 0.017041 \\
\hline Cerebellum White Matter & 1.319 & 0.194795 & 0.417 & 0.079 & 0.071875 \\
\hline Precentral Gyrus & 1.196 & 0.238644 & 0.378 & 0.124 & 0.022441 \\
\hline Medial Postcentral Gyrus & 1.061 & 0.294904 & 0.336 & 0.070 & 0.090174 \\
\hline Brainstem & -0.875 & 0.386852 & 0.277 & 0.005 & 0.640175 \\
\hline Cerebellar Vermal Lobules VI-VII & 0.699 & 0.488487 & 0.221 & 0.024 & 0.325448 \\
\hline Cerebellar Vermal Lobules VIII-X & 0.617 & 0.541029 & 0.195 & 0.015 & 0.434774 \\
\hline Cerebellar Vermal Lobules I-V & 0.218 & 0.828189 & 0.069 & 0.016 & 0.430517 \\
\hline Globus Pallidus & -0.212 & 0.832833 & 0.067 & 0.001 & 0.860265 \\
\hline Calcarine Cortex & 0.198 & 0.843964 & 0.063 & 0.013 & 0.473158 \\
\hline
\end{tabular}

Notes: $\uparrow$ rows are sorted based on the $p$ values for the t-test; ${ }^{*} p<0.000793$ Bonferroni corrected for multiple comparison; $d$ represents Cohen's d effect size; MMSE: mini mental state examination 
Table 3. Summary of the independent-sample t-tests comparing volumetric data between the participants with Alzheimer's disease and healthy controls and the correlation of the data with MMSE scores using volBrain method.

\begin{tabular}{llllll}
\hline & \multicolumn{3}{l}{ Comparison between groups } & \multicolumn{2}{l}{ Correlation with MMSE } \\
\cline { 2 - 6 } Brain Area & $\boldsymbol{t}$ & $\boldsymbol{p} \uparrow$ & $\boldsymbol{d}$ & $\boldsymbol{r}^{2}$ & $\boldsymbol{p}$ \\
\hline Amygdala & 10.217 & $<0.000001^{*}$ & 3.231 & 0.428 & $0.000001^{*}$ \\
Hippocampus & 6.58 & $<0.000001^{*}$ & 2.081 & 0.256 & $0.000395^{*}$ \\
Accumbens & 5.813 & $0.000001^{*}$ & 1.838 & 0.339 & $0.000027^{*}$ \\
Thalamus & 4.422 & $0.000065^{*}$ & 1.398 & 0.317 & $0.000057^{*}$ \\
Caudate & 4.149 & $0.000154^{*}$ & 1.312 & 0.169 & 0.005091 \\
Cerebellum & 2.063 & 0.045216 & 0.652 & 0.094 & 0.041135 \\
Globus Pallidus & -1.103 & 0.276245 & 0.349 & 0.026 & 0.287427 \\
Putamen & 0.846 & 0.402030 & 0.268 & 0.019 & 0.366267 \\
\hline
\end{tabular}

Notes: $\uparrow$ rows are sorted based on the $p$ values for the t-test; ${ }^{*} p<0.0038$ Bonferroni corrected for multiple comparison; $d$ represents Cohen's d effect size; MMSE: mini mental state examination; 
Table 4. Summary of the independent-sample t-tests comparing volumetric data between the participants with Alzheimer's disease and healthy controls and the correlation of the data with MMSE scores using BrainSuite segmentation method.

\begin{tabular}{|c|c|c|c|c|c|}
\hline \multirow[b]{2}{*}{ Brain Area } & \multicolumn{3}{|c|}{ Comparison between groups } & \multicolumn{2}{|c|}{ Correlation with MMSE } \\
\hline & $\mathbf{t}$ & $\mathbf{p} \uparrow$ & d & $\mathbf{r} 2$ & $\mathbf{p}$ \\
\hline Inferior Temporal Gyrus & 7.245 & $<0.000001^{*}$ & 2.184 & 0.677 & $0.000001 *$ \\
\hline Middle Temporal Gyrus & 4.738 & $0.000029 *$ & 1.429 & 0.697 & $<0.000001 *$ \\
\hline Third Ventricle & -4.354 & $0.000094 *$ & 1.313 & -0.522 & $0.000468 *$ \\
\hline Superior Temporal Gyrus & 3.944 & $0.000323 *$ & 1.189 & 0.507 & $0.000722 *$ \\
\hline Supramarginal Gyrus & 3.698 & $0.000668 *$ & 1.115 & 0.509 & $0.000683^{*}$ \\
\hline Angular Gyrus & 3.632 & $0.000809 *$ & 1.095 & 0.52 & $0.000498^{*}$ \\
\hline Middle Occipital Gyrus & 3.543 & 0.001043 & 1.068 & 0.536 & $0.000303 *$ \\
\hline Pars Opercularis & 2.958 & 0.005237 & 0.892 & 0.45 & 0.003137 \\
\hline Inferior Occipital Gyrus & 2.663 & 0.011206 & 0.803 & 0.383 & 0.013402 \\
\hline Accumbens & -2.64 & 0.011866 & 0.796 & -0.481 & 0.001457 \\
\hline Superior Parietal Gyrus & 2.534 & 0.015392 & 0.764 & 0.434 & 0.004538 \\
\hline Superior Colliculus & -2.532 & 0.015481 & 0.763 & -0.338 & 0.030512 \\
\hline Parahippocampal Gyrus & 2.447 & 0.019029 & 0.738 & 0.428 & 0.005294 \\
\hline Cingulate Gyrus & -2.374 & 0.022629 & 0.716 & -0.356 & 0.022147 \\
\hline Fusiform Gyrus & 2.269 & 0.028864 & 0.684 & 0.155 & 0.334311 \\
\hline Insula & 2.133 & 0.039251 & 0.643 & 0.255 & 0.107583 \\
\hline Globus Pallidus & -1.995 & 0.053041 & 0.602 & -0.231 & 0.146663 \\
\hline Cerebellum & 1.979 & 0.054949 & 0.597 & 0.191 & 0.232289 \\
\hline Basal Forebrain & -1.909 & 0.063590 & 0.576 & -0.243 & 0.126172 \\
\hline Anterior Orbito-Frontal Gyrus & 1.856 & 0.070973 & 0.560 & 0.234 & 0.140158 \\
\hline Subcallosal Gyrus & -1.847 & 0.072330 & 0.557 & -0.207 & 0.194909 \\
\hline Pars Orbitalis & 1.82 & 0.076458 & 0.549 & 0.275 & 0.081327 \\
\hline Lingual Gyrus & -1.809 & 0.078196 & 0.545 & -0.316 & 0.043952 \\
\hline Middle Frontal Gyrus & 1.807 & 0.078496 & 0.545 & 0.284 & 0.071904 \\
\hline Lateral Geniculate Nucleus & 1.78 & 0.082929 & 0.537 & 0.253 & 0.109970 \\
\hline Middle Orbito-Frontal Gyrus & 1.744 & 0.089100 & 0.526 & 0.142 & 0.374241 \\
\hline Temporal Pole & 1.591 & 0.119752 & 0.480 & 0.022 & 0.892295 \\
\hline Post-Central Gyrus & 1.501 & 0.141345 & 0.453 & 0.315 & 0.044533 \\
\hline Hippocampus & -1.241 & 0.221958 & 0.374 & -0.162 & 0.311495 \\
\hline Transverse Temporal Gyrus & 1.138 & 0.261864 & 0.343 & 0.268 & 0.090858 \\
\hline Transvers Frontal Gyrus & 1.099 & 0.278660 & 0.331 & 0.272 & 0.085861 \\
\hline
\end{tabular}




\begin{tabular}{llllll}
\hline Thalamus & 0.968 & 0.339074 & 0.292 & 0.087 & 0.586936 \\
Inferior Colliculus & 0.94 & 0.352768 & 0.283 & 0.102 & 0.526091 \\
Pars Triangularis & 0.911 & 0.367812 & 0.275 & 0.216 & 0.174328 \\
Claustrum & 0.87 & 0.389785 & 0.262 & 0.163 & 0.308227 \\
Caudate & -0.863 & 0.393478 & 0.260 & -0.122 & 0.448542 \\
Pre-Central Gyrus & 0.859 & 0.395408 & 0.259 & 0.242 & 0.128116 \\
Paracentral Lobule & 0.858 & 0.395895 & 0.259 & 0.128 & 0.424284 \\
Superior Occipital Gyrus & 0.858 & 0.395943 & 0.259 & 0.169 & 0.291590 \\
Cuneus & 0.843 & 0.404153 & 0.254 & 0.106 & 0.510235 \\
Putamen & 0.653 & 0.517714 & 0.197 & -0.001 & 0.995473 \\
Lateral Orbitofrontal Gyrus & -0.548 & 0.586738 & 0.165 & -0.071 & 0.658410 \\
Gyrus Rectus & -0.516 & 0.608533 & 0.156 & -0.123 & 0.442668 \\
Medial Geniculate Nucleus & -0.505 & 0.616072 & 0.152 & -0.122 & 0.448980 \\
Mamillary Body & 0.492 & 0.625193 & 0.148 & 0.125 & 0.436896 \\
Precuneus & -0.49 & 0.627078 & 0.148 & -0.007 & 0.967476 \\
Posterior Orbito-Frontal Gyrus & 0.445 & 0.658856 & 0.134 & 0.02 & 0.901794 \\
Brainstem & 0.209 & 0.835439 & 0.063 & -0.015 & 0.925512 \\
Superior Frontal Gyrus & 0.202 & 0.840699 & 0.061 & 0.132 & 0.411455 \\
Amygdala & 0.145 & 0.885584 & 0.044 & -0.109 & 0.497327 \\
\hline
\end{tabular}

Notes: $\uparrow$ rows are sorted based on the $p$ values for the $\mathrm{t}$-test; ${ }^{*} p<0.001000$ Bonferroni corrected for multiple comparison; $d$ represents Cohen's d effect size; MMSE: mini mental state examination 
Table 5. Summary of the independent-sample t-tests comparing volumetric data between the participants with Alzheimer's disease and healthy controls and the correlation of the data with MMSE scores using HIPS segmentation method.

\begin{tabular}{llllll}
\hline & \multicolumn{3}{l}{ Comparison between groups } & \multicolumn{2}{l}{ Correlation with MMSE } \\
\cline { 2 - 6 } Brain Area & $\boldsymbol{t}$ & $\boldsymbol{p} \uparrow$ & $\boldsymbol{d}$ & $\boldsymbol{r}^{2}$ & $\boldsymbol{p}$ \\
\hline SR-SL-SM & 8.990 & $<0.000001^{*}$ & 2.843 & 0.393 & $0.000004^{*}$ \\
Hippocampus & 8.619 & $<0.000001^{*}$ & 2.726 & 0.388 & $0.000005^{*}$ \\
CA4/Dentate Gyrus & 8.248 & $<0.000001^{*}$ & 2.608 & 0.402 & $0.000003^{*}$ \\
CA1 & 6.308 & $<0.000001^{*}$ & 1.995 & 0.256 & $0.000389^{*}$ \\
Subiculum & 5.121 & $0.000007^{*}$ & 1.619 & 0.229 & $0.000873^{*}$ \\
CA2-CA3 & 5.025 & $0.000009^{*}$ & 1.589 & 0.288 & $0.000142^{*}$ \\
\hline
\end{tabular}

Notes: $\uparrow$ rows are sorted based on the $p$ values for the t-test; ${ }^{*} p<0.008333$ Bonferroni corrected for multiple comparison; $d$ represents Cohen's d effect size; MMSE: mini mental state examination; SRSL-SM: strata radiatum/lacunosum/moleculare 
Table 6. Correlation of the size of common brain areas reported by the three segmentation methods

\begin{tabular}{|c|c|c|c|c|c|}
\hline & \multirow[b]{2}{*}{ Brain Area } & \multicolumn{2}{|l|}{ CAT } & \multicolumn{2}{|c|}{ volBrain } \\
\hline & & $r^{2}$ & $p$ & $r^{2}$ & $p$ \\
\hline \multirow[t]{7}{*}{ BrainSuite } & Accumbens & -0.227 & 0.159813 & -0.277 & 0.078996 \\
\hline & Amygdala & 0.240 & 0.136249 & 0.029 & 0.858460 \\
\hline & Caudate & 0.169 & 0.298015 & 0.090 & 0.577545 \\
\hline & Globus Pallidus & -0.118 & 0.469153 & 0.184 & 0.249666 \\
\hline & Hippocampus & -0.162 & 0.318747 & -0.275 & 0.081327 \\
\hline & Putamen & 0.328 & 0.039077 & -0.186 & 0.243349 \\
\hline & Thalamus & 0.188 & 0.245249 & 0.177 & 0.268448 \\
\hline \multirow[t]{7}{*}{ volBrain } & Accumbens & 0.633 & $0.000007 *$ & & \\
\hline & Amygdala & 0.632 & $0.000007 *$ & & \\
\hline & Caudate & 0.470 & $0.001678 *$ & & \\
\hline & Globus Pallidus & -0.245 & 0.118543 & & \\
\hline & Hippocampus & 0.637 & $0.000006^{*}$ & & \\
\hline & Putamen & 0.020 & 0.898315 & & \\
\hline & Thalamus & 0.541 & $0.000214^{*}$ & & \\
\hline
\end{tabular}

Notes: ${ }^{*} p<0.002380$ Bonferroni corrected for multiple comparison 


\section{Figures' Caption}

Figure 1. Processing pipeline for volBrain and HIPS adapted from Manjón and Coupé (2016) ${ }^{34}$ under the terms of the Creative Commons Attribution License (CC BY).

Figure 2. Processing pipeline for CAT

Figure 3. Processing pipeline for BrainSuite

Figure 4. Subcortical structures in an AD patient and a HC participant using CAT segmentation method. The histograms show the volume of each brain area

Figure 5. Cerebellum MRI-T1 brain segmentation in an AD patient and a HC participant using volBrain segmentation method.

Figure 6. Cerebellum MRI-T1 brain segmentation in an AD patient and a HC participant using BrainSuite segmentation method.

Figure 7. Left and right hippocampus subfield segmentation in an $\mathrm{AD}$ patient and a $\mathrm{HC}$ participant 
bioRxiv preprint doi: https:/doi.org/10 1101/2020 08 18.256321: this version posted August 19, 2020. The copyright holder for this preprint (which was not certified by peer review) is the author/funder, who has granted bioRxiv a license to display the preprint in perpetuity. It is made available under aCC-BY-NC-ND 4.0 International license.

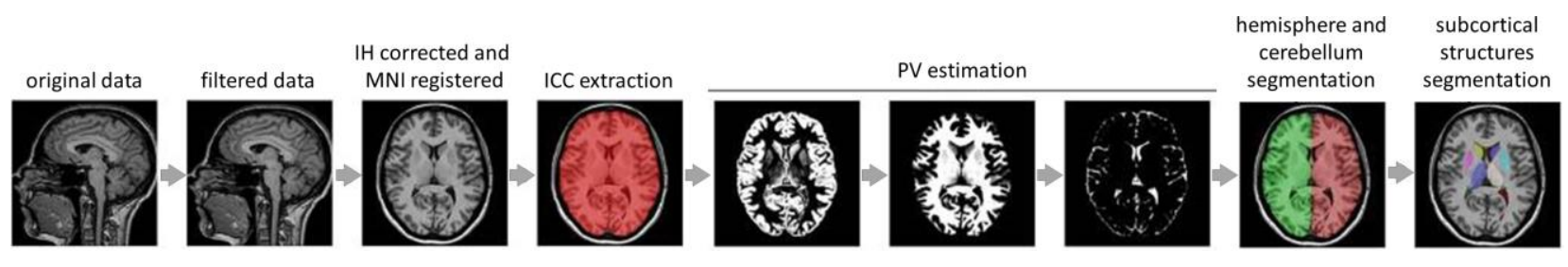

Figure 1. Processing pipeline for volBrain and HIPS adapted from Manjón and Coupé (2016) ${ }^{34}$ under the terms of the Creative Commons Attribution License (CC BY). 
bioRxiv preprint doi: https://doi.org/10.1101/2020 08 18.256321; this version posted August 19, 2020. The copyright holder for this preprint (which was not certified by peer review) is the author/funder, who has granted bioRxiv a license to display the preprint in perpetuity. It is made available under aCC-BY-NC-ND 4.0 International license.

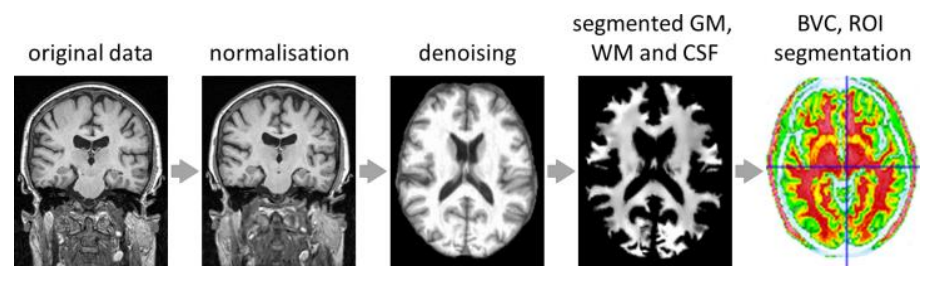

Figure 2. Processing pipeline for CAT. 
bioRxiv preprint doi: https://doi.org/10.1101/2020 08 18.256321; this version posted August 19, 2020. The copyright holder for this preprint (which was not certified by peer review) is the author/funder, who has granted bioRxiv a license to display the preprint in perpetuity. It is made available under aCC-BY-NC-ND 4.0 International license.

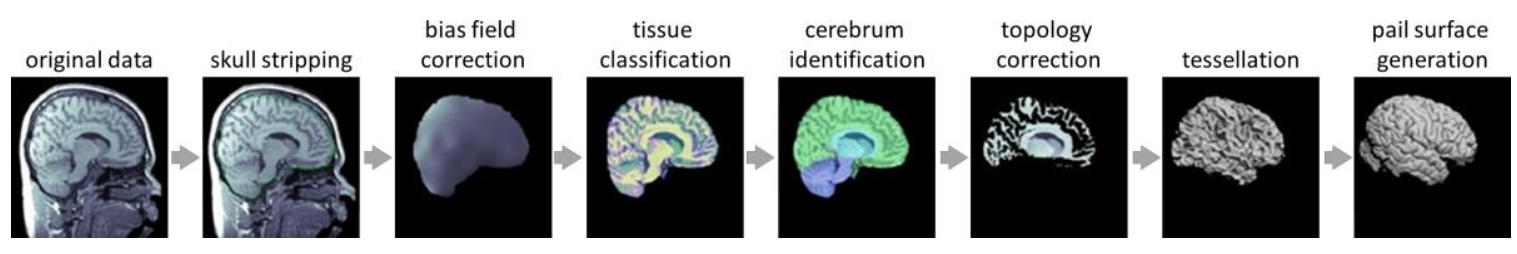

Figure 3. Processing pipeline for BrainSuite. 


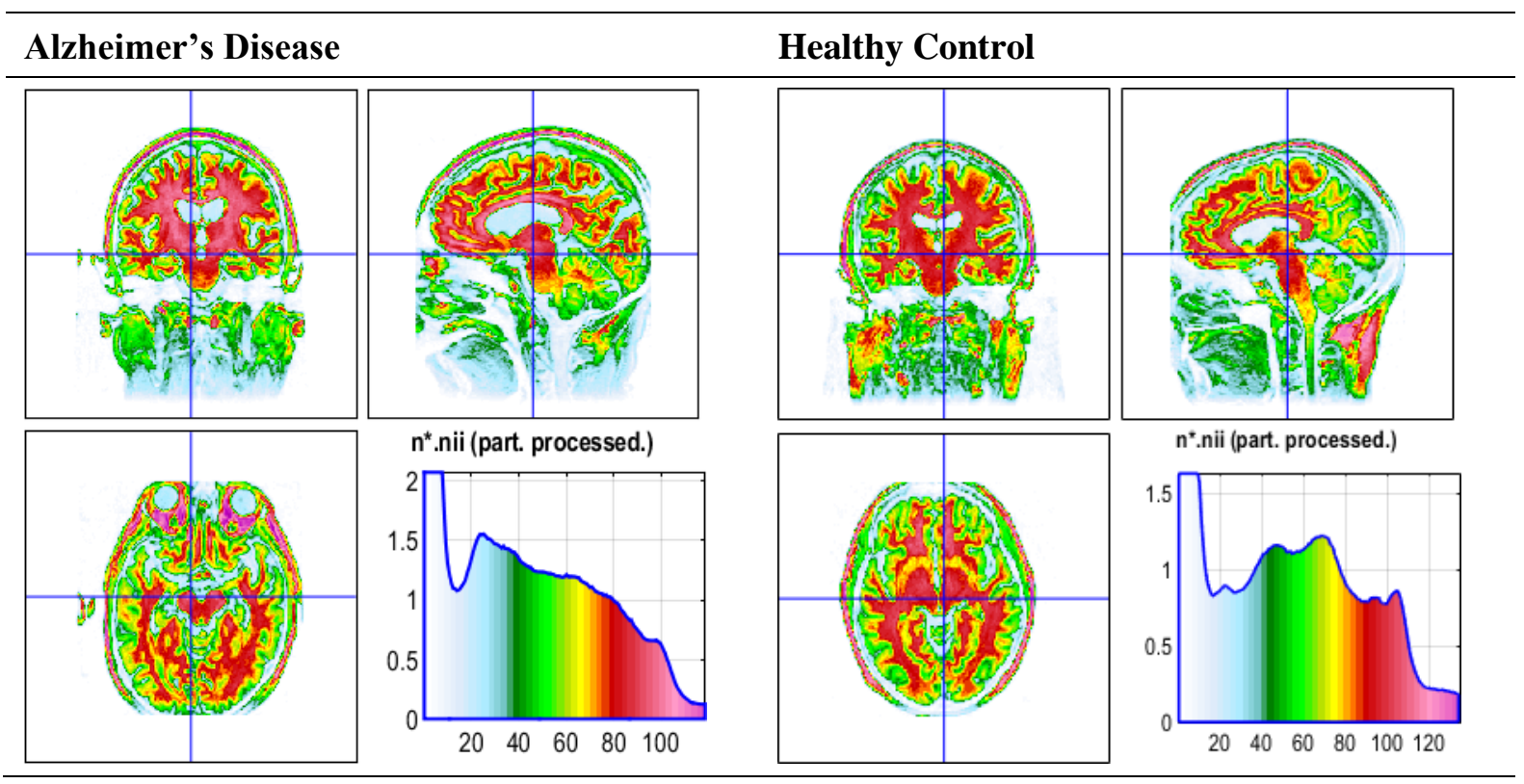

Figure 4. Subcortical structures in an AD patient and a HC participant using CAT segmentation method.

The histograms show the volume of each brain area. 
bioRxiv preprint doi: https://doi org/10.1101/2020.08.18.256321; this version posted August 19, 2020. The copyright holder for this preprint (which was not certified by peer review) is the author/funder, who has granted bioRxiv a license to display the preprint in perpetuity. It is made available under aCC-BY-NC-ND 4.0 International license.

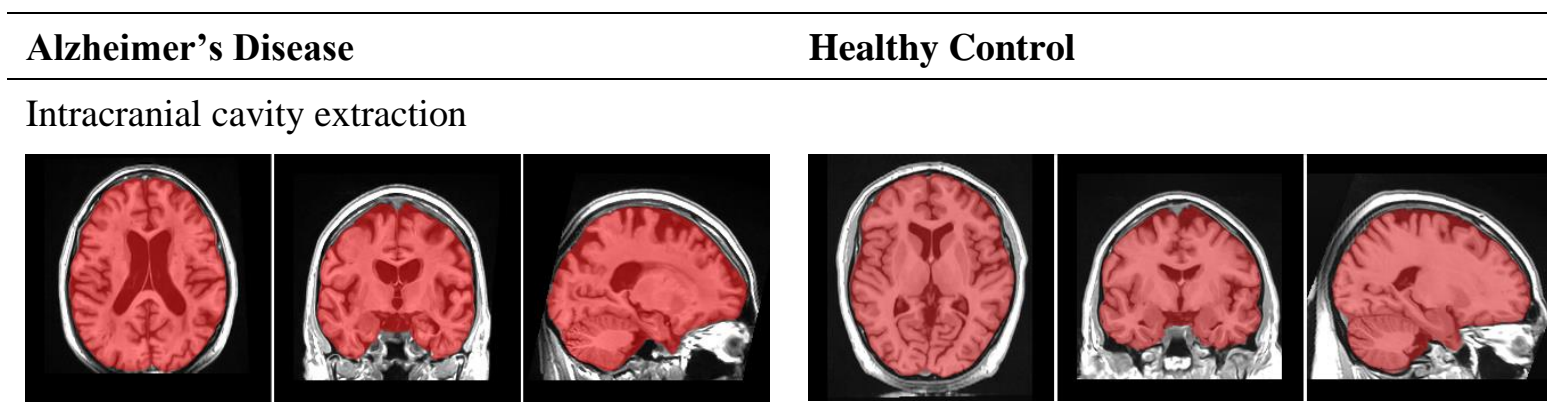

Tissue classification
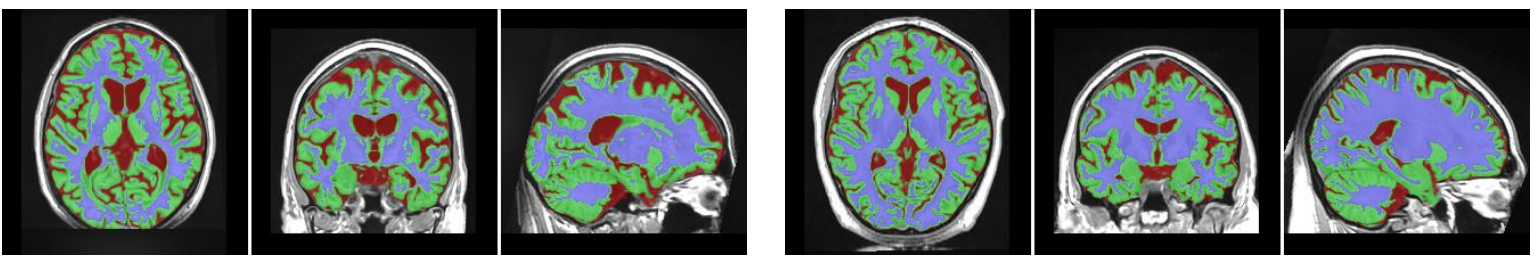

Macrostructures
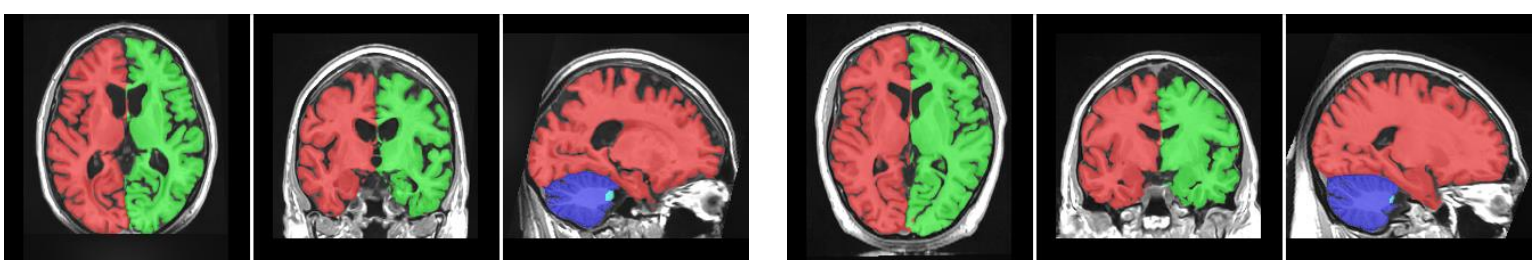

Subcortical Structures
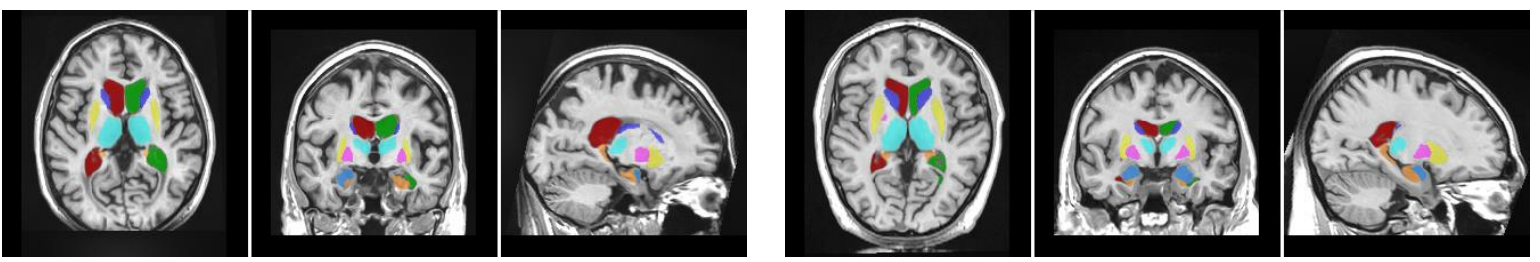

Figure 5. Cerebellum MRI-T1 brain segmentation in an AD patient and a HC participant using volBrain segmentation method. 
bioRxiv preprint doi: $h$ ttps://doi.org/10.1101/2020.08.18 256321: this version posted August 19, 2020. The copyright holder for this preprint (which was not certified by peer review) is the author/funder, who has granted bioRxiv a license to display the preprint in perpetuity. It is made available under aCC-BY-NC-ND 4.0 International license.

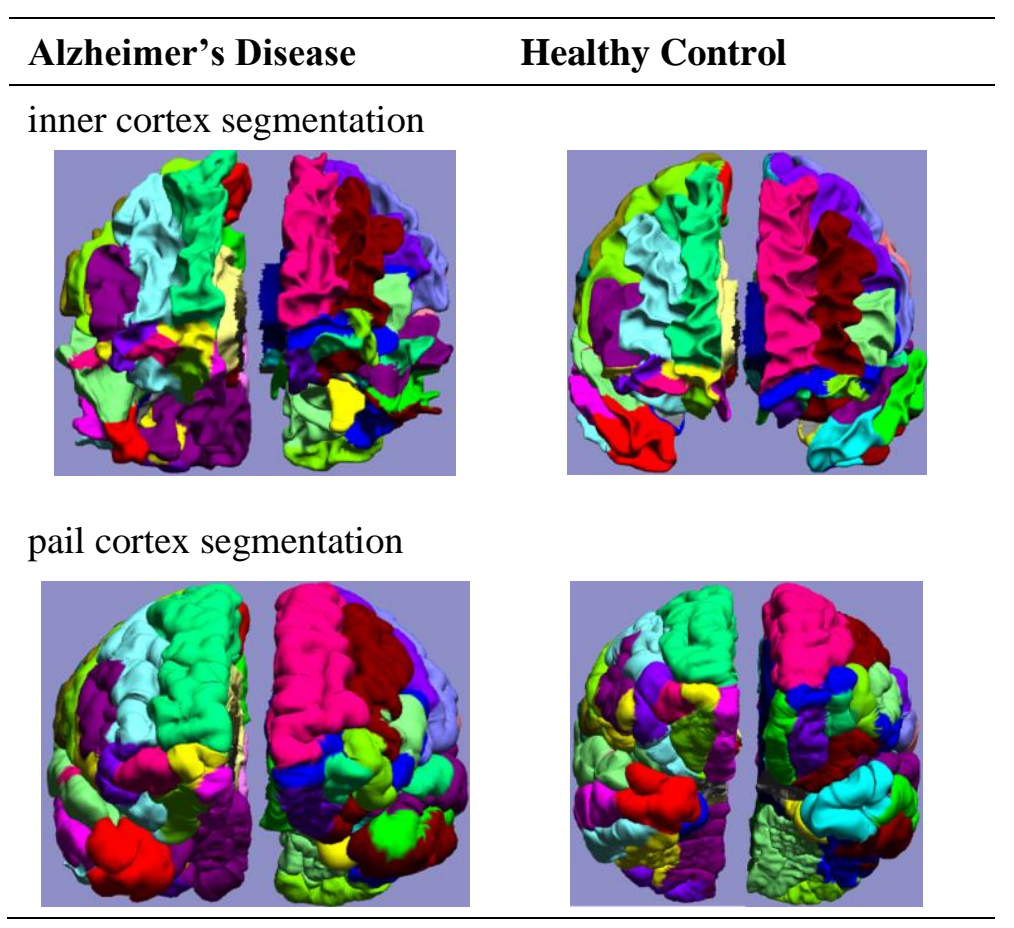

Figure 6. Cerebellum MRI-T1 brain segmentation in an AD patient and a HC participant using BrainSuite segmentation method. 


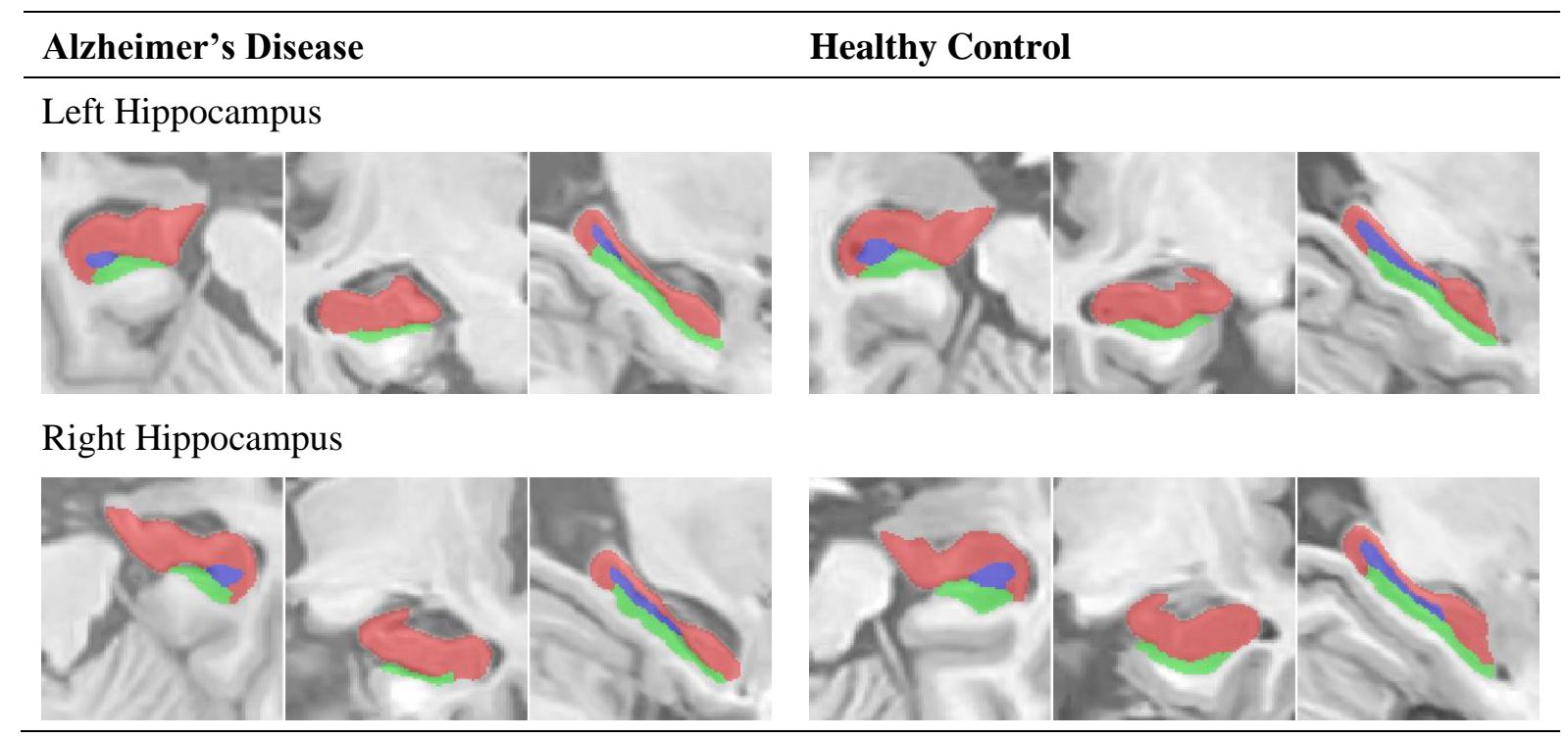

Figure 7. Left and right hippocampus subfield segmentation in an AD patient and a HC participant using HIPS segmentation method. 